\title{
FALSO RESIDUO POSTMICCIONAL DIAGNOSTICADO POR VIDEOURODINAMIA
}

\author{
M. MUSQUERA FELIP, C. ERRANDO SMET, M. PRADOS SAAVEDRA, \\ P. ARAÑÓ BERTRÁN, H. VILLAVICENCIO MAVRICH.
}

Unidad de Urología Funcional y Femenina. Servicio de Urología. Fundació Puigvert. Barcelona.

Actas Urol Esp. 28 (10): 792-795, 2004

\section{RESUMEN}

FALSO RESIDUO POSTMICCIONAL DIAGNOSTICADO POR VIDEOURODINAMIA

Presentamos un caso de un paciente varón joven diagnosticado de reflujo vésico ureteral bilateral. En el estudio urodinámico se objetiva una posible disinergia vesico-esfinteriana no neurógena, con gran residuo postmiccional. Tras tratamiento con biofeedback se soluciona la disinergia, persistiendo el gran residuo. El estudio con videourodinamia permitió diagnosticar la presencia de un falso residuo postmiccional causado por el vaciamiento ureteral del reflujo bilateral masivo.

PALABRAS CLAVE: Videourodinamia. Reflujo vesico ureteral. Sindrome de Hinmann.

\section{ABSTRACT}

FALSE POSTVOID RESIDUAL VOLUME DIAGNOSED BY VIDEOURODYNAMICS

We present a case report of a young male patient, with a bilateral vesico renal reflux. The urodynamic study findings suggested the possibility of a non-neurogenic bladder-external spincter dissinergya producing a valuable residual volume. After biofeedback treatment, the dissinergia disappeaed, but residual volume persisted. The videourodynamic assessment allowed us the accurate diagnosis of a false residual volume, produced by the voiding of the refluxed urine from the ureters into the bladder.

KEY WORDS: Videourodynamics. Vesico ureteral reflux. Hinmaris Syndrome.

$\mathrm{E}^{1}$ síndrome de Hinmann (síndrome de micción no coordinada, "no neurogenic neurogenic bladder"), es una disfunción que consiste en una incoordinación entre la contracción del detrusor y la relajación del esfínter urinario esquelético, produciéndose una obstrucción funcional por contracciones esfinterianas durante la micción. La causa es idiopática aunque se sugiere como etiología un mal aprendizaje de la micción durante edades precoces de la vida. Clínicamente puede aparecer urgencia, incontinencia, infección de orina de repetición y eucopresis. En ocasiones se acompaña de signos de uropatía obstructiva crónica.
El diagnóstico requiere del estudio urodinámico, y se basa en la demostración de actividad esfinteriana durante la contracción voluntaria del detrusor durante la micción.

La videourodinamia es una modalidad de estudio urodinámico que nos permite realizar al mismo tiempo un estudio completo (con electromiografía(EMG)), y una cistografía de relleno con uretrografía miccional. En el caso del síndrome de micción no coordinada permite diagnosticar al mismo tiempo la disinergia vesico esfinteriana, su repercusión sobre el flujo miccional, así como la presencia de reflujo vesicoureteral con el que en ocasiones se asocia. 
Presentamos un caso de un paciente de 18 años de edad diagnosticado inicialmente de síndrome de Hinmann y residuo postmiccional elevado, que mediante la videourodinamia se diagnosticó de falso residuo por reflujo vesicoureteral masivo y por lo tanto diagnostico inicial erróneo.

\section{CASO CLÍNICO}

Varón de 18 años de edad con antecedentes de micosis fungoide (síndrome linfoproliferativo). Una ecografía reno-vesical practicada de forma rutinaria informó de normalidad renal, con imagen hipoecoica trigonal compatible con megaureter. Mediante urografía intravenosa se confirmó megauréter bilateral, vejiga de gran tamaño con importante residuo post-miccional. El paciente aportaba un informe de cistouretrografía retrógrada y miccional que informaba de uretra sin alteraciones y reflujo bilateral grado 1 .

Acude a nuestro centro donde se solicita renograma MAG3 que descarta obstrucción de tramo urinario superior. Se realiza una flujometría fisiológica que muestra un flujo máximo de 27,3 $\mathrm{ml} / \mathrm{seg}$ (volumen miccional: $281 \mathrm{ml}$ ) con residuo post-miccional de $100 \mathrm{ml}$. La morfología de la curva de flujo es gausiana aunque con mellado terminal (Fig. 1).

La cistometría descarta patología de la fase de llenado, (capacidad cistométrica máxima: $649 \mathrm{ml}$, acomodación: $108 \mathrm{ml} / \mathrm{cmH}_{2} \mathrm{O}$ ). En el estudio de presión-flujo se observa micción por contracción voluntaria y sostenida del detrusor, con flujo

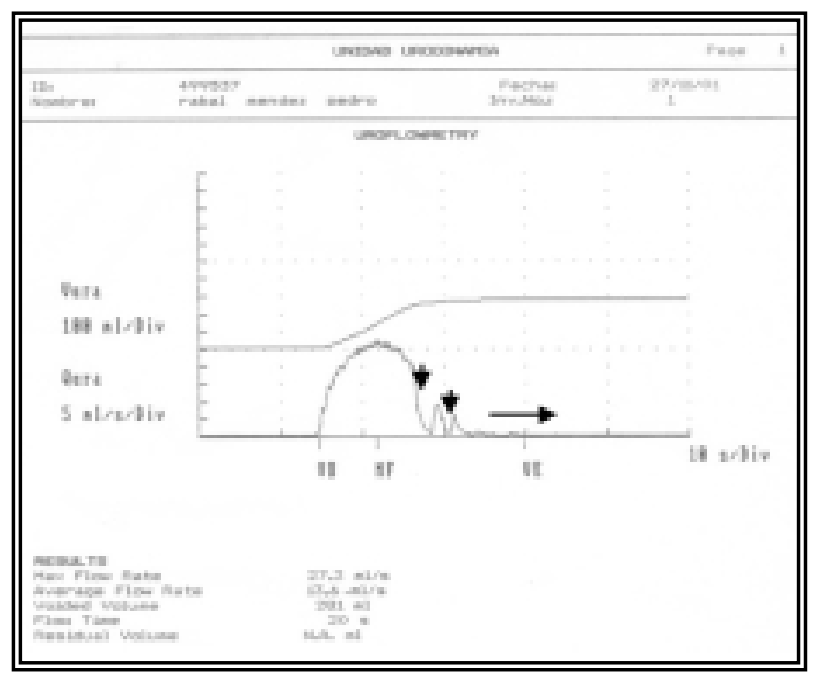

FIGURA 1 máximo de $22 \mathrm{ml} / \mathrm{seg}$ y una presión del detrusor de $33 \mathrm{~cm} \mathrm{de} \mathrm{H}_{2} \mathrm{O}$ (resistencia uretral: 0,06). Al final de la micción se observa un aumento de la actividad electromiográfica del esfinter estriado, que condiciona la caída súbita del flujo miccional con un incremento simultáneo de la presión del detrusor (Fig. 2). Residuo post-miccional de 230 $\mathrm{ml}$ (Fig. 3). Se realizó un diario miccional con medición seriada de residuos, objetivándose micciones de entre $250-400 \mathrm{ml}$, con residuos persistentes comprendidos entre 150 a $250 \mathrm{ml}$.

Con la orientación diagnóstica de disinergia no neurógena (síndrome de Hinmann, síndrome de micción disfuncional, non-neurogenic neurogenic bladder), se indicó tratamiento mediante autosondaje intermitente para control de residuos y

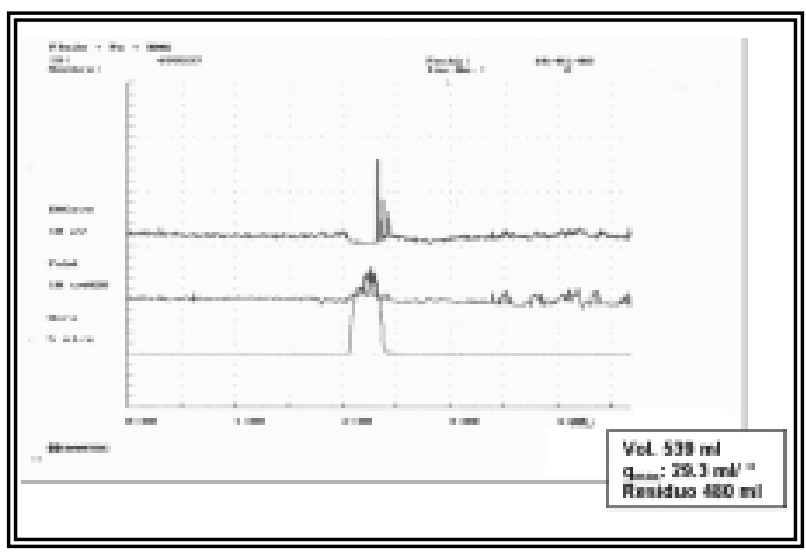

FIGURA 2

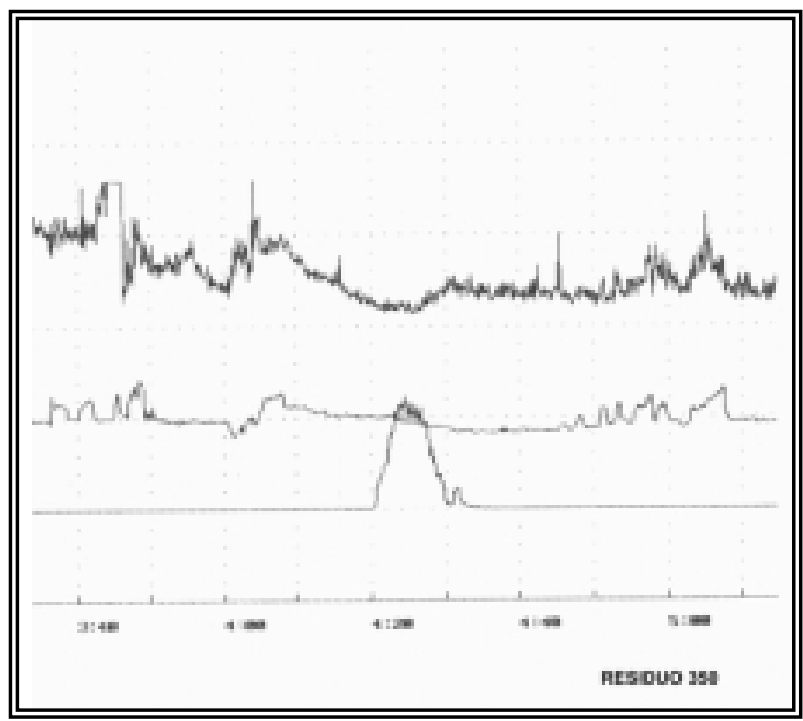

FIGURA 3 
biofeedback miccional para corregir la incoordinación del esfínter esquelético. Tras 3 sesiones se evidenció la desaparición de la actividad enfinteriana durante la micción.(Fig. 3).

Dada la persistencia de residuo elevado $(350 \mathrm{ml})$ se decidió realizar una videourodinamia con intención de aclarar la discordancia clínico-urodinámica. Durante la fase de llenado se observó reflujo vésicoureteral izquierdo pasivo, en ausencia de hiperactividad vesical. En la fase de vaciado se objetivó el vaciado completo de la vejiga y simultáneamente reflujo bilateral masivo (Figs. 4 y 5). Tras la micción, se apreció el vaciado ureteral en vejiga causante del falso residuo (Fig. 6).

Se indicó cirugía antirreflujo, practicándose Cohen bilateral, estando el paciente en la actualidad asintomático.

\section{DISCUSIÓN}

El síndrome de Hinmann es una disfunción del esfinter estriado que consiste en la contracción simultánea del esfinter estriado y del detrusor durante la fase de vaciado, causando una obstrucción funcional de tipo intermitente o continuo. Puede presentar signos de uropatía obstructiva como vejiga de paredes trabeculadas, residuo post-miccional elevado, uréterohidronefrosis $\mathrm{y}$ reflujo vésicoureteral, cuadro semejante a una vejiga neurógena pero en ausencia de patología neurológica. Clínicamente pueden presentar flujo miccional en escopetazo o interrumpido, urgencia miccional, incontinencia e infecciones de repetición ${ }^{1}$. Es importante descartar el síndrome de Hinmann en niños que presentan escape diurno ${ }^{2}$, infecciones urinarias de repetición, residuo post miccional y sobretodo en aquellos que presentan reflujo vesicoureteral ${ }^{3,4}$.

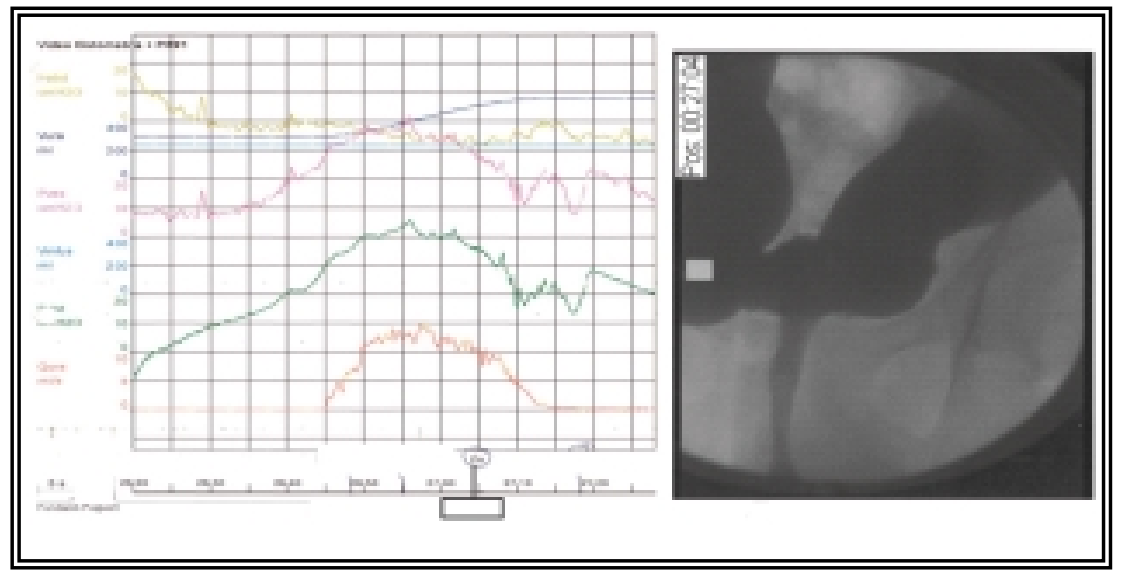

FIGURA 4

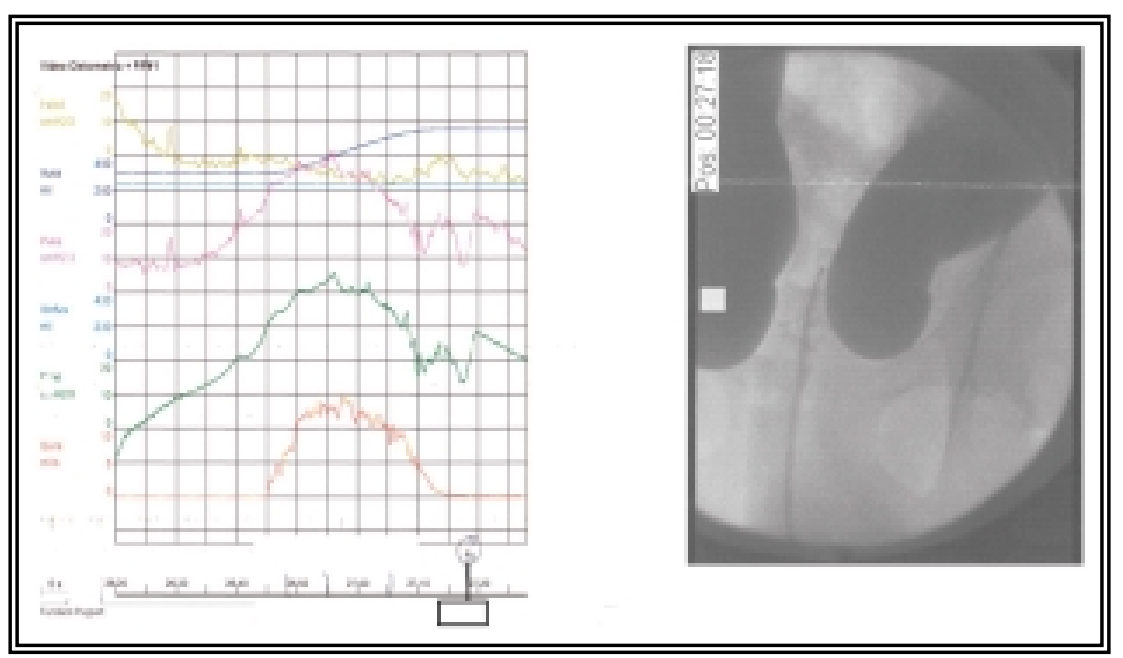

FIGURA 5
El diagnóstico se basa en la demostración mediante estudio urodinámico de contracciones incoordinadas del esfinter estriado durante la contracción voluntaria del detrusor.

El tratamiento de elección es el biofeedback ${ }^{5,6}$. Consiste en una forma de aprendizaje en la que el paciente se coloca un circuito de retroalimentación, donde procesos fisiológicos inconscientes se hacen aparentes mediante una señal, que primero es susceptible de reconocer y más tarde modificar mediante esta técnica terapéutica ${ }^{3,5,6}$. En caso de existir residuo importante como el caso que presentamos se puede realizar autocateterismo intermitente, con el fin de evitar posibles complicaciones infecciosas.

En este caso, el biofeedback fue eficaz, objetivándose mediante electromiografía de control la desaparición de la actividad esfinteriana durante el vaciado. Sin embargo, el hallazgo de un aparente residuo post-miccional por 


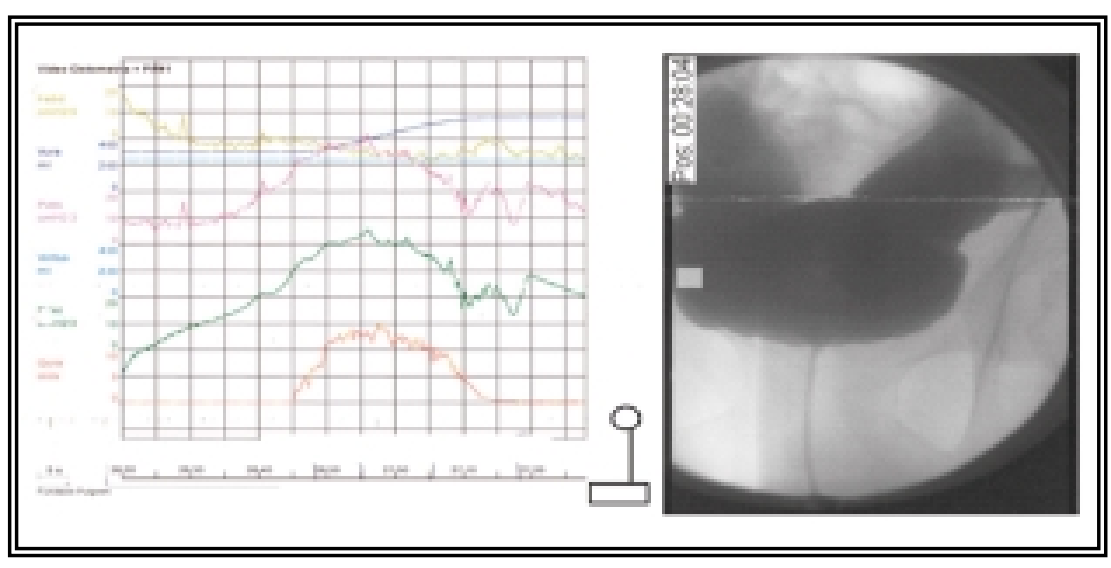

FIGURA 6 la fase de llenado y vaciado, y su relación con existencia de reflujo, morfología vesical, y alteraciones del funcionalismo vésico-esfinteriano durante el vaciado $^{8,9}$.

Consideramos que los pacientes con residuo elevado y reflujo de alto grado, constituyen una indicacion para la videourodinamia, dado que nos permite descartar la presencia de orina procedente del reflujo, como causa de falso residuo postmiccional. sondaje, nos condujo a realizar una videourodinamia. Dicha exploración permitió demostrar que tras el biofeedback, la micción era sinérgica, y conseguía el vaciado de la vejiga, ya que el aparente residuo medido por sondaje, correspondía realmente a orina que había refluido a ambos uréteres durante la fase de llenado y durante la contracción del detrusor. Ello permitió realizar la indicación de cirugía antirreflujo, dado que se demostró que no existía hiperactividad del detrusor como causa del reflujo.

La videourodinamia ofrece una completa información funcional y anatómica, aunque existe controversia en sus indicaciones debido a la infraestructura que requiere y su coste-efectividad, no siendo en la actualidad una prueba de uso rutinario. Entre las indicaciones más aceptadas están:

1. Sospecha de obstrucción infravesical en la mujer, dado que permite valorar flujo, contractilidad del detrusor, apertura del cuello durante la micción, localización anatómica de la obstrucción, existencia de divertículos uretrales... ${ }^{7}$.

2. Obstrucción infravesical en el varón joven. Permite realizar el diagnóstico de disinergia lisa o de cuello vesical (observándose la no apertura del cuello vesical durante la micción con detrusor contráctil y flujo bajo.

3. Vejiga neurógena, dado que no siempre existe correlación entre la evaluación neurológica, la clínica miccional y la disfunción vesical subyacente. La videourodinamia permite estudiar las combinaciones entre las alteraciones de

\section{REFERENCIAS}

1. Hoebeke P, Van Laecke E, Van Camp C, Raes A, Van de Walle J. One thousand video-urodynamic studies in children with non-neurogenic bladder sphincter dysfunction. BJU international 2001; 87:575-580.

2. Hinman F, Baumann FW. Vesical and ureteral damage from voiding dysfunction in boys without neurologic or obstructive disease. The Journal of Urology 1973; 109:(727-732).

3. Van Gool JD, Vijverberg MAW, de Jong TPVM. Functional daytime incontinence: clinical and urodynamic assessment. Clinical and urodyinamic assessment. Scand J Urol Nephrol 1992; 52:S141.

4. Hoebeke P, Vande Walle J, Everaert K, Van Laecke E, Van Gool JD. Assessment of lower urinary tract disfunction in children with non-neuropathic bladder sphincter dysfunction. Eur Urol 1999;35:57-69.

5. Rapariz MA. Reeducación esfinteriana en el síndrome de micción no coordinada. Arch. Esp. de Urol. 1997; 50(6):626632.

6. Medina López RA, Pena Outeiriño JMạ , León Dueñas E, Leal López A. El biofeedback como alternativa terapéutica en la micción no coordinada. Actas. Urol. Esp. 1998; 22(7):616619.

7. Hann-Chorng Kuo. Videourodynamic results after pubovaginal sling procedure for stress urinary incontinence. Urology 1999 Nov; 54(5): 802-807.

8. Johnston, L B; Borzyskowski, M.Bladder dysfunction and neurological disability at presentation in closed spina bifida. Archives of disease in Childhood 1998; 79(1):33-38.

9. Mevorach RA,. Bogaert GA, Baskin LS, Lazzaretti CC, Edwards MSB, Kogan BA. Lower urinary tract function in ambulatory children with spina bifida. British Journal of Urology 1996; 77: 593-596.

Dra. M. Musquera Felip

Servicio de Urología

Fundació Puigvert

C/ Cartagena, 340-350

08025 Barcelona

(Trabajo recibido el 17 noviembre de 2003) 